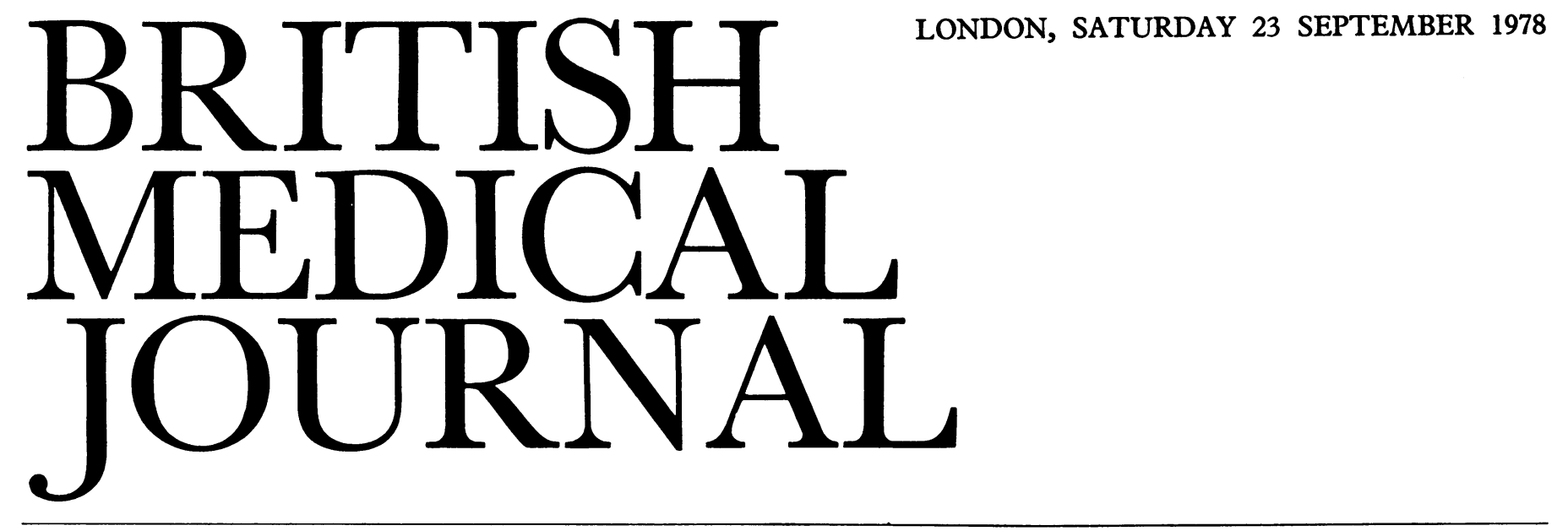

\title{
Poliomyelitis vaccines: killed or live?
}

To all the recent controversies about immunisation there has been added, in the case of poliomyelitis, a revival of interest in the choice between live oral and inactivated parenteral vaccine. Some type of poliovaccine is unavoidably needed to prevent recurrent epidemics of poliomyelitis in countries which have crossed the threshold of development and improved hygiene (often corresponding ${ }^{1}$ to an infant mortality rate of under 75 per 100000 live births). Even in developing countries in the pre-epidemic stage where only sporadic cases of infantile paralysis occur and all those over the age of 4 years are immune from natural exposure poliomyelitis can inflict a serious burden of crippling illness-7 per 100000 schoolchildren, equivalent to an annual attack rate of 28 per 100000 in a recent survey in Ghana. ${ }^{2} 3$

For economy and simplicity most countries use oral (Sabin type) vaccines, though experience in Sweden, Finland, and the Netherlands show that inactivated (Salk type) vaccine can also be effective-provided in both cases that the vaccines and their delivery are closely controlled for efficacy and safety in the field as well as in the laboratory. An immunisation rate as near $100 \%$ as possible should be aimed at, since the penalty of paralytic poliomyelitis is rapidly exacted from groups and individuals unimmunised, whether from neglect and ignorance or from religious prejudice or other personal beliefs. Recent examples of this in highly immunised countries using inactivated vaccine were outbreaks in a natural lifestyle commune in Sweden ${ }^{4}$ and in strictly religious communities in the Netherlands. ${ }^{56}$ Sporadic cases and small outbreaks in recent years in Britain and the United States, where oral vaccines have mainly been used, have particularly affected the less-wellimmunised areas. Omission of an unsatisfactory type 3 component of oral vaccine in Poland in 1966 was followed promptly by a type 3 epidemic ${ }^{7}$ in 1968.

As an aid to rational choice between the two available types of vaccine the World Health Organisation has recently published a detailed review by J L Melnick. ${ }^{8}$ Inactivated vaccine confers humoral immunity in a satisfactory proportion of vaccinees if given in sufficient dosage (this protects the nervous system against viraemic invasion if poliovirus infects the gut; it may also reduce secondary pharyngeal invasion from the gut and minimise ororespiratory spread in conditions of good hygiene where the faecal-oral route is less important). The inactivated vaccine can be combined with bacterial antigens as part of routine paediatric immunisation. Because it contains no living virus, potential mutation and reversion to virulence cannot occur; and this absence of living virus permits its use in immunodeficient or immunosuppressed individuals and their households. Inactivated virus has, indeed, greatly reduced the spread of poliovirus in countries where it has been efficiently used and might prove useful in some tropical areas where live vaccine has failed to "take" in a proportion of young infants.

Against these advantages must be set the unsatisfactory proportion of vaccinees developing antibody after three doses in several studies (probably owing to inadequate antigenicity, especially in some early commercial vaccines). Furthermore, booster doses are usually required to maintain detectable antibody titres (though, again, this may not always be true if antigen dosage is adequate); and inactivated vaccines do not induce local (intestinal) immunity and therefore have little effect on inhibiting faecal-oral circulation of the virus in the population (in fact, evidence of some local immunity from inactivated vaccine has been reported ${ }^{9}$ but this is unlikely to be as effective as the immunity produced by live vaccine virus). The currently licensed inactivated vaccines are made in monkey kidney cell cultures, and there is a growing world scarcity of monkeys. Finally, should a failure in virus inactivation occur the virulent seed viruses might lead to tragedy (fortunately the techniques of inactivation and test methods are much better understood now than at the time of the "Cutter incident"). In future it might be wise to prepare inactivated vaccines from attenuated seed viruses such as those used for live vaccine.

A similar balance sheet can be drawn up for live attenuated vaccine. Like natural infection, it confers both humoral and local intestinal immunity (intestinal immunity to reinfection is neither absolute nor permanent but does tend to block the spread of virus circulating in the community). This induced immunity may give lifelong protection and additional doses in later life should not be required except to plug possible gaps in the trivalent immunity. Indeed, since intestinal immunity prevents vaccine virus from establishing itself no anamnestic "booster" effect is produced, though this can be achieved by injecting inactivated vaccine into children with pre-existing antibody. ${ }^{10}$ Live vaccine induces antibody quickly in a large proportion of recipients; it is more acceptable, and does not require highly trained personnel. The potency of stabilised vaccine can be well maintained in field conditions with little refrigeration and no freezing. In epidemics the rapid infection of the alimentary tract by vaccine virus interferes with spread of the epidemic poliovirus. The vaccine is relatively inexpensive both to produce and to administer; and it can readily be prepared in human cell cultures. 
On the other hand, live vaccines may mutate; rarely they may revert to virulence during growth in vaccinees and so cause paralytic poliomyelitis in recipients or their contacts. (For that reason all susceptible family members should be vaccinated simultaneously to minimise the chance of reverted virus being transmitted to non-immune close contacts.) These risks should not be exaggerated: a five-year study by a special committee of $\mathrm{WHO}^{11}$ showed attack rates in recipients varying from 0 to 2 per million doses of vaccine distributed (one per 4 million doses adminstered in England and Wales ${ }^{12}$ ). Most of those affected were children under 5 years old infected with type 3 virus; the rates in contacts ranged from 0.1 to 0.6 per million doses, except in two high-incidence countries which had used high-passage seed virus without continuous control. Most of these contact infections were by type 2 virus, often in non-immune parents of recently vaccinated infants in countries which, like Britain, vaccinate throughout the year. In countries which concentrate polio vaccination into short campaigns most cases were in children under 5 years old. These are maximal estimates of risk, based on circumstantial evidence: no laboratory test can yet identify unequivocally whether a particular strain of poliovirus has originated from vaccine or otherwise. The potential of live vaccine progeny to spread to close contacts, formerly regarded as an advantage, is nowadays considered a disadvantage. This unlicensed progeny virus may also spread to persons in the community who have not agreed to be vaccinated.

In some countries with warm climates oral vaccine fails to induce immunity in a satisfactorily high proportion of recipients. This failure may be due to interference by hyperendemic enteroviruses or more probably to immunoglobulinlike inhibitors in infants' saliva, ${ }^{13}$ which may be combated by simultaneous administration of equine antibody to human gammaglobulin or by giving repeated doses of vaccine. Finally, the live vaccine is contraindicated in persons with immunodeficiency diseases or on immunosuppressive treatment, and in their household contacts.

The WHO report confirmed that “. . . poliomyelitis vaccines (oral) made from the Sabin attenuated strains are among the safest vaccines in use today." There is no adequate reason for countries using the inexpensive and efficient oral vaccine to change to the inactivated vaccine. Equally there appears to be no reason for the opposite change by countries which can afford to use inactivated vaccine of high quality on the scale necessary to prevent poliomyelitis. But the price of freedom from polio is eternal vigilance: whatever the type of vaccine in use, it must be the subject of continued laboratory and epidemiological control. Surveillance of the population must include antibody surveys to detect gaps in immunity which may indicate failure of the vaccine or of its uptake by the population.

\footnotetext{
${ }^{1}$ Paul, J R, Bulletin of the World Health Organisation, 1958, 19, 747.

2 Nicholas, D D, et al, British Medical fournal, 1977, 1, 1009.

${ }^{3}$ Ofosu-Amaah, S, Kratzer, J H, and Nicholas, D D, British Medical fournal, 1977, 1, 1012.

${ }^{4}$ World Health Organisation, Weekly Epidemiological Record, 1977, 52, 138.

5 Bijkerk, H, et al, Nederlands Tijdschrift voor Geneeskunde, 1972, 116, 549.

6 World Health Organisation, Weekly Epidemiological Record, 1978, 53, 187.

7 World Health Organisation, Regional Office for Europe, Communicable Diseases: Methods of Surveillance. Report on a seminar, The Hague, 1969. Copenhagen, World Health Organisation, 1969.

${ }^{8}$ Melnick, J L, Bulletin of the World Health Organisation, 1978, 56, 21.

${ }_{9}^{9}$ Dick, G W A, et al, British Medical fournal, 1961, 2, 266.

${ }_{10}$ Reid, D, et al, Lancet, 1969, 1, 564.

11 Assaad, F, et al, Bulletin of the World Health Organisation, 1976, 53, 319.

${ }_{12}$ Miller, D, Reid, D, and Diamond, J R, Public Health, London, 1970, 84, 265.

${ }^{13}$ Domok, I, Bulletin of the World Health Organisation, 1974, 51, 333.
}

\section{Nutrition and the patient with cancer}

Nutritional depletion is a common hallmark of patients with $\stackrel{.}{\hookrightarrow}$ cancer. Many factors may contribute to the characteristic $\overrightarrow{\vec{F}}$ cachexia. Clinically, there is loss of body protein and fat, $\overrightarrow{0}$ anaemia, electrolyte disturbances, and weakness and debility. 을 Anorexia is a major problem, and many patients seem satisfied $\frac{\bar{m}}{\sigma}$ by a little intake. Emotional problems such as depression may $\underset{\complement}{\complement}$

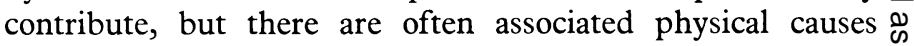
such as ulceration and infection of the mouth, nausea, and $\vec{\theta}$ vomiting, and there may be abnormalities of taste-in particular, blunting of the sense of sweetness, heightened sensi- $\vec{\omega}$ tivity to bitterness, and aversion to meat. ${ }^{1}$ Whatever its cause, $\frac{\partial}{\partial}$ the management of the anorexia requires psychological support, good preparation of food, and the elimination of oral $\frac{N}{\sigma}$ infection if the patient is to eat enough. Mechanical obstruction of the gastrointestinal tract or the biliary or pancreatic ducts $\vec{\Phi}$ and fistula formation may also contribute to the cachexia by $\stackrel{\infty}{\mathrm{f}}$ limiting intake or absorption; while tumour necrosis, ulcera- $\mathrm{O}$ tion, associated infection, and haemorrhage all result in excessive loss of protein.

Some specific changes are seen in animals and patients with $\mathbb{D}$ cancer but not in starvation. Calman and McAllister ${ }^{2}$ reported $\frac{\vec{D}}{D}$ a fall in the co-enzyme A content of liver and muscle within $\bar{Z}$ 24 hours of implanting tumours in animals, with the implica- $\frac{\mathbb{D}}{-}$ tion that $\beta$-oxidation of fatty acids is impaired. Warnold $\vec{\theta}$ et $a l^{3}$ compared energy balance in patients with cancer with $\propto$ controls free from malignant disease. Though they found no 0 significant difference in energy intake, they confirmed Pratt's $\mathrm{s}^{4} \sum_{\Sigma}^{\circ}$ results in animal studies and found energy expenditure and $\overline{0}$ resting metabolic rate were significantly increased in the patients with cancer. This increased energy expenditure may $\stackrel{\varnothing}{\stackrel{D}{2}}$ be an additional cause of cachexia. After curative surgery $\overrightarrow{\overrightarrow{0}}$ energy expenditure returned to the control values.

At a recent symposium at the University of Surrey special emphasis was placed on the vitamin requirements of patients with cancer. Vitamin A deficiency is associated with tumours of the gastrointestinal tract, nasopharynx, and lung. ${ }^{5}$ Since vitamin A may be important in maintaining adequate serum $\frac{5}{3}$ concentrations of non-specific opsonins ${ }^{6}$ and is an effective immunostimulant, ${ }^{7}$ depletion may depress the immune res- $\frac{0}{3}$ ponse. Low leucocyte concentrations of vitamin $\mathrm{C}$ have been described in patients with bone metastases from carcinoma of the breast-a finding associated with depression of phago- $\sigma$ cytosis by neutrophils, ${ }^{8}$ reduced macrophage migration, ${ }^{9} \frac{D}{O}$ increased incidence of wound dehiscence, ${ }^{10}$ and alterations in drug metabolism.

Surgery, chemotherapy, and radiotherapy, singly or in $\mathrm{\omega}^{\circ}$ combination, may further compromise the nutritional state

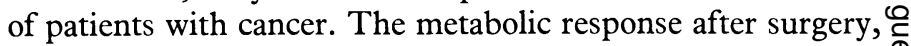
with its associated loss of lean tissue, is well documented, but $\stackrel{\Phi}{\rightleftharpoons}$ further depletion may be associated with cancer chemotherapy. Some chemotherapeutic agents are analogues of essential nutrients: methotrexate, for example, is an antagonist of folic $\stackrel{\Phi}{\Phi}$ acid. A sprue-like syndrome with the characteristic malabsorp- $\mathbb{\mathbb { D }}$ tion may be produced by agents such as 5-fluouracil, which induces morphological and biochemical changes in the gastro- $\delta$ intestinal mucosa: typically the height of the villi is reduced and intestinal dipeptidases are depleted, so impairing assimila-

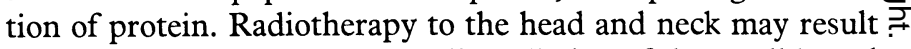
in stomatitis and dysphagia, while radiation of the small bowel may cause oedema and endarteritis of small blood vessels, 